Communications in Physics, Vol. 28, No. 3 (2018), pp. 191-200

DOI:10.15625/0868-3166/28/3/12359

\title{
A COMPACT DEVICE MODEL FOR NANOPARTICLE-ORGANIC MEMORY TRANSISTOR'S CHARACTERIZATION
}

\author{
MAI VAN HUY ${ }^{a, \dagger}$ OLIVIER BICHLER ${ }^{b}$, CHRISTIAN GAMRAT $^{b}$ YANNICK VIERO $^{c}$, \\ FABIEN ALIBART ${ }^{c}$ AND DOMINIQUE VUILLAUME ${ }^{c}$
}

${ }^{a}$ Le Quy Don Technical University,

234 Hoang Quoc Viet, Bac Tu Liem, Hanoi, Vietnam

${ }^{b}$ CEA LIST, 91191 Gif-sur-Yvette, France

${ }^{c}$ Institute for Electronics Microelectronics and Nanotechnology (IEMN),

CNRS, Univ. of Lille, CS 60069, 59652 Villeneuve d'Ascq, France

${ }^{\dagger} E$-mail: vanhuy.mai@gmail.com

Received 23 April 2018

Accepted for publication 26 June 2018

Published 31 August 2018

\begin{abstract}
Neuromorphic electronic devices have recently been a candidate for new computing architecture associated with innovative nanotechnologies. A report of the characterization of Nanoparticle organic memory transistor (NOMFET) introduced a similar behavior to a biological spiking synapse in neural networks. In this paper, a refinement model based on the extracted parameters including a hybrid NOMFET/CMOS neuromorphic computing circuit and architecture of synapse to neuron interface by characterizing transistor - memory and the temporal dynamic function is presented. A compact EKV model refinement serves as a link between nanotechnology process and circuit design for novel CMOS devices.
\end{abstract}

Keywords: dynamics, hybrid integrated circuit, modeling, nanotechnology, neural networks, synapse-like nanodevices, EKV model.

Classification numbers: 07.50.Ek, 84.30.-r .

\section{INTRODUCTION}

Neuromorphic electronics appears as a promising computing architecture to replace the traditional Von Neumann architecture. The spiking neural network (SNN) is a network model, which uses relative spike timing for information coding, inspires from how the brain works, to (C)2018 Vietnam Academy of Science and Technology 
allow fast and efficient information processing for complex tasks, such as recognition or classification, involving a new class of nanodevices - synapse transistor [1]. A nanoparticle organic memory field-effect transistor (NOMFET) made of pentacene and gold nanoparticles deposited in the gate channel was demonstrated to act as an organic memory-transistor device [2]. Its most interesting behavior - emulation of biological spiking synapse- is due to charge storage in the nanoparticles [3]. It can be programmed to work as a facilitating or depressing synapse, and exhibits short-term plasticity (STP) for dynamical processing of spikes. The physical mechanism of NOMFET has been reported in $[3,4]$.

A simple functional model based on a few physical equations and a number of experimental parameters has already been developed, simulating the dynamic charge and discharge behavior of the NOMFET as a spiking synaptic device [4]. It was the first model that could be used to simulate hybrid neuromorphic circuits using NOMFET as synapses and CMOS as neurons. However, it was restricted to a single device bias configuration and did not include transistor field effect modeling. In this paper, we propose a full compact model for the NOMFET, modeling both its charge-discharge dynamic and the transistor field effect based on the EKV compact and fast transistor model, which was already successfully used to simulate some Fet-Nano devices $[4,5]$. The nanoparticles charge dynamic is introduced by modulating the transistor threshold voltage with the nanoparticles charge. The model is fitted with both transistor experimental characterizations and temporal synaptic-like dynamic characterizations.

After a brief description of the device physics and experimental characterizations in a first part, we present the model building and refinement, followed by the presentation of the fitted results which compare the model with the experimental data.

\section{EXPERIMENTAL MEASUREMENTS}

The NOMFET described in Fig. 1 composed of three terminals as a conventional metaloxide-semiconductor field-effect transistor [4]. It is fabricated by using a bottom-gate electrode configuration, where the p+ -highly doped silicon gate is covered with $200 \mathrm{~nm}$ thick of silicon dioxide $\left(\mathrm{SiO}_{2}\right)$ by dry thermal oxidation. The gate is fabricated by E-beam lithography Ti/Au (thickness $2 / 18 \mathrm{~nm}$ ). A $60 \mathrm{~nm} \mathrm{SiO}_{2}$ thin film is deposited by PECVD. The source (S) and drain (D) terminals are $\mathrm{Ti} / \mathrm{Au}(20 / 180 \mathrm{~nm})$ electrodes with inter-electrode gap in the range of $5 \mu \mathrm{m}$. Gold nanoparticles (NPs, diameters of $10 \mathrm{~nm}$ ) are immobilized on the surface of the inter-electrode gap by surface chemistry before pentacene deposition $(20 \mathrm{~nm})$. The pentacene is a p-type organic semiconductor. The conduction between the drain and the source is modulated by charging and discharging the holes in channel, which is created in the thin film at the interface with $\mathrm{SiO}_{2}$. By modulating the conductivity of its channel through the charging of nano-particles embedded into the organic semi-conducting channel, the NOMFET is behaving like a memristive device [1]. In pulse regime, it exhibits many behaviors of a dynamic synapse such as potentiation and depression $[6,7]$ and, also showing a cumulative effect.

In previous experiments [2,3], the gate and drain were connected together forming a dipole with voltage applied cross. For the new technological devices, the gate and drain are separated. A voltage is applied on the gate forming an electric field perpendicular to the channel. This allows having a better distribution of the NP charging effect when compared to the previous horizontal

field across the channel. By applying a series of pulses on the gate, the gold nanoparticles are 
charged holes to the interface between the pentacene and the $\mathrm{SiO}_{2}$. And we measure the current between the drain and the source.

Two types of characterizations were conducted in the following: transistor characterization $I_{D S}=f\left(V_{D S}\right)$ and $I_{D S}=f\left(V_{G S}\right)$ when nanoparticles are fully charged and fully discharged, and temporal charge dynamic $I_{D S}=f(t)$ for various initial charging state of the device.

For transistor characterization, we studied the influence of the gate voltage on the channel current after writing or erasing, we applied a $-30 \mathrm{~V}$ voltage during $30 \mathrm{~s}$ on the gate electrode for writing and of $30 \mathrm{~V}$ during $30 \mathrm{~s}$ for erasing while the source and drain electrodes were grounded. The $I_{D S}$ current was then measured as a function of gate voltage $V_{G S}$, which is swiped from $0 \rightarrow$ $-30 \mathrm{~V}$ and $0 \rightarrow 30 \mathrm{~V}$. The experimental measurement is shown in dots curves in Fig. 7. For the measurement $I_{D S}=f\left(V_{D S}\right)$, we applied a $-30 \mathrm{~V}$ voltage during $30 \mathrm{~s}$ on the gate for a write and $30 \mathrm{~V}$ voltage during $30 \mathrm{~s}$ for a erase, going measurement the current $I_{D S}$ for a swiped voltage $0 \rightarrow-30 \mathrm{~V}$ on the drain while the gate is applied $-30 \mathrm{~V}$.

For the temporal charge dynamic, we measured the evolution of the device current as a function of time, by applying a voltage pulse on the gate with the drain-source voltage is $V_{D S}=$ $-15 \mathrm{~V}$ for measuring the current of charge and discharge after each applied pulse and also $-15 \mathrm{~V}$ on the gate.

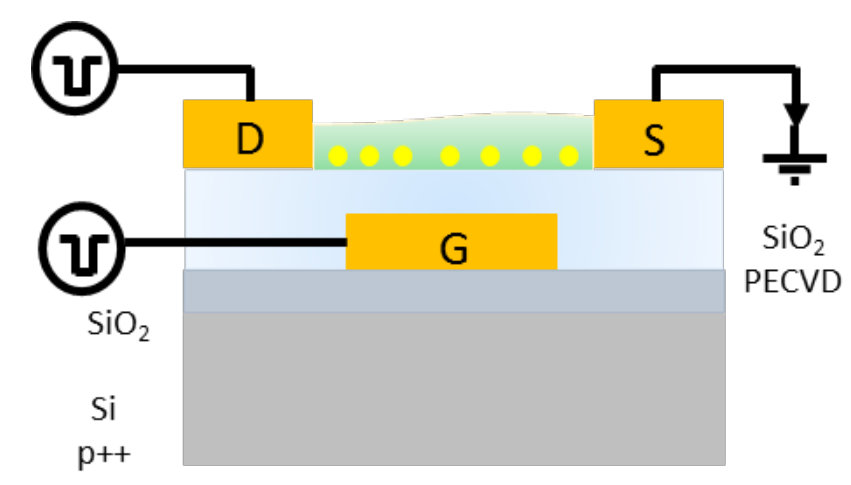

Fig. 1. Schematic of the physical structure of the NOMFET transistor and our experimental setup [3].

In our experiment, the organic pentacene is a p-type semiconductor, when a negative voltage is applied on the gate the holes formed from drain to source are trapped by the gold nanoparticles, resulting in a charging current that flows from source to drain. When the gate voltage becomes null or positive, the holes are removed from the gold nanoparticles and the device is discharged.

\section{EKV MODEL}

In MOSFET, with the aggressive downscaling of CMOS technologies, it is required a model which is enabled for the design and the optimization of new low-power and low-voltage analog and RF circuit where most transistor were operating in the weak and moderate inversion regime [8]. For an accurate implicit formulation of the current, the characteristics of MOSFETs is exploited by MOS modelling [9], and an analytical EKV MOS transistor model [10]. The EKV MOS transistor 
model introduced a powerful concept of inversion factor as the main transistor design parameter showing a capability to design and sizing new circuits.

\section{III.1. EKV based model}

We use the compact EKV model [11], in which the current $I_{D S}$ through the transistor represents the difference between two current components: the forward current component $I_{\text {for }}$, which depends on the source voltage $V_{S}$ (Eq. (2)) and the reverse current component $I_{\text {rev }}$, which depends on the drain voltage $V_{D}$ (Eq. (3)). This model allows a simple compact equation describing a behavior that changes smoothly from an exponential relationship towards a smooth saturation. The model uses several parameters: the off current $\mathrm{I}_{o f f}$, the slope factor $n$, the threshold voltage $V_{T 0}$, the linear/quadratic $\theta$ and the overall scale $I_{S}$ (Fig. 2).

The current $I_{D S}$ between two terminals is expressed by the following equation:

$$
I_{D S}=I_{S} \frac{I_{f o r}-I_{r e v}}{1+\theta V_{p}}+I_{o f f},
$$

where:

$$
\begin{aligned}
& I_{\text {for }}=\left[\ln \left(1+e^{\left(v_{p}+v_{S}\right) / 2 V_{t}}\right)\right]^{2}, \\
& I_{\text {rev }}=\left[\ln \left(1+e^{\left(v_{p}+v_{D}\right) / 2 V_{t}}\right)\right]^{2},
\end{aligned}
$$

With the thermal voltage $V_{t}=k_{B} \cdot T / e$ ( $k_{B}$ is the Boltzmann constant, $T$ is the ambient temperature, $e$ is the elementary charge).

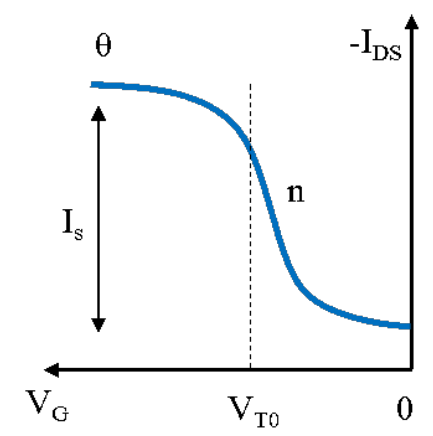

$\mathrm{I}_{\mathrm{DS}}$ : drain current in log scale

$I_{\text {off: }}$ off current

$\mathrm{n}$ : slope factor

$\mathrm{V}_{\mathrm{T} 0}$ : threshold voltage

$\theta:$ linear/quadratic

$\mathrm{I}_{\mathrm{s}}$ : overall scale

$\mathrm{V}_{\mathrm{G}}$ : gate voltage

Fig. 2. EKV model.

The pinch-off voltage $V_{P}$ is defined as the channel voltage for which the inversion charge becomes zero under strong inversion assumption, and is calculated a linear function of the gate voltage $V_{G}$ [12], the threshold voltage $V_{T 0}$, and the slope factor $n$ [5]. For NOMFET, the charge stored in the $\mathrm{NP}_{s}$ induce a shift in the threshold voltage [13], which is estimated by the number of charge holes in gold $\mathrm{NP}_{s} \quad q$ and the oxide capacitance $C_{o x}$, hence the pinch-off voltage $V_{p}$ is calculated by:

$$
V_{p}=-\frac{1}{n}\left(V_{G}-V_{T O}+\frac{q}{C_{o x}}\right)
$$


In a first time, the charge of nanoparticles is estimated with a simple first-order charge equation depending on the charge relaxation time constant $\tau_{C}$, the residual charge $q_{0}$ at $V_{G}=0$, and the capacitance of gold nanoparticle $C_{N P}$, given by:

$$
q=\left(q-q_{0}+C_{N P} V_{N P}\right) e^{-\frac{d t}{\tau_{C}}}+q_{0}-C_{N P} V_{N P}
$$

The charging voltage $V_{N P}$ for the gold nanoparticle is estimated by the voltage difference between the gate and the voltage at the center of the channel as following

$$
V_{N P}=V_{G}-\frac{V_{S}+V_{D}}{2}
$$

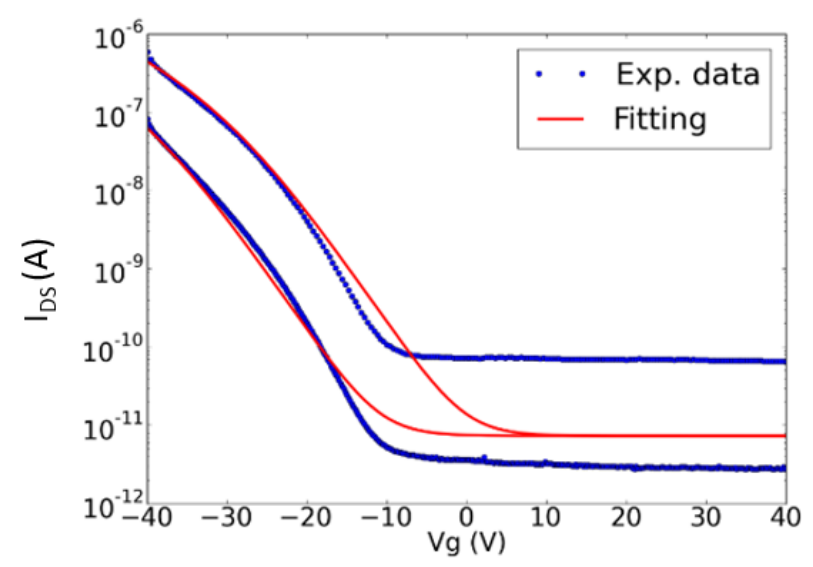

(a)

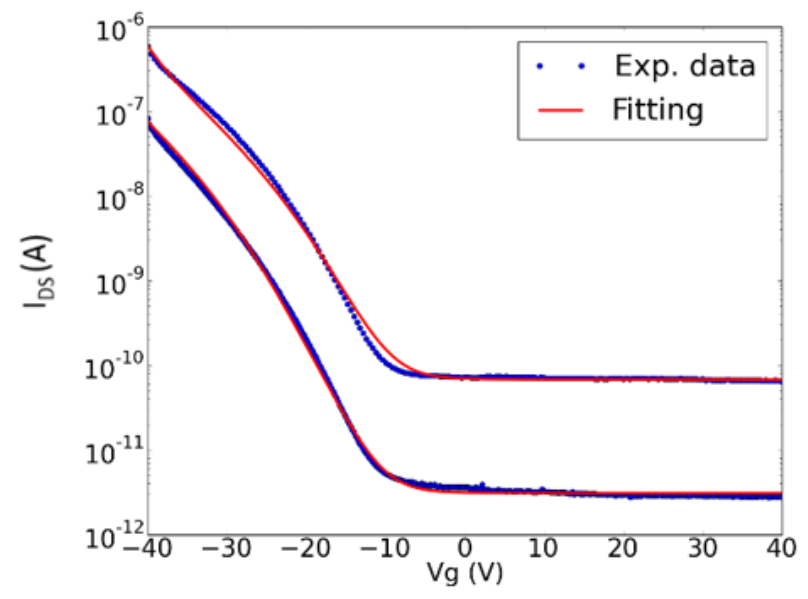

(b)

Fig. 3. The influence of $I_{\text {off }}$ on the fitting: (a) without $S$; (b) with $S$.

In first simulation, we have characterized the NOMFET transistor by the current between drain and source in function of gate voltage after a write of $-30 \mathrm{~V}$ and a read of $+30 \mathrm{~V}$ as show the solid curves in Fig. 3 (a). These curves of model are not in agreement with the experimental results (dotted curves) in the positive voltage part. When $V_{G S}<V_{t}$ the MOSFET is in the offstate, however, an undesirable leakage current can flow between the drain and the source called the subthreshold current. This is the main contributor to the MOSFET off-state current $I_{\text {off }}$. The 
subthreshold current is proportional to $\exp \left(q\left(V_{g s}-V_{t}\right) / \eta k T\right)$. At room temperature, the function $\exp \left(q . V_{g s} / k T\right)$ changes by 10 for every $60 \mathrm{mV}$ change in $V_{g s}$. Using subthreshold swing $S=$ $\eta .60 \mathrm{mV} \frac{T}{300 \mathrm{~K}}$ and $I_{\text {off }}$ is measured at $V_{g s}=0$ and $V_{d s}=V_{d d}$, therefore the off current modulation can be modeled by $10^{-\frac{V_{t}}{S}}[14]$.

The experimental data show that $I_{o f f}$ is modulated by the threshold voltage and hence the NPs charge. It is caused by the influence of $I_{o f f}$ for which the current is not the same after a negative voltage of write and read of transistor-memory. For this solution, we use the $S$ parameter to permit separate the current in the saturated situation as shown in Fig 3 (b), there is a good agreement between two curves from the model and the measured data.

\section{III.2. Refinement fitting of temporal dynamic of NOMFET}

In the following, we focus on the dynamic, temporal, behavior of the EKV model. The first simulation of the EKV model showing the simple first-order charge equation for NPs did not fit very well with experimental curves as can be seen in Fig. 4. Two problems can be observed:

1. We see a shift between the model and experiments in the NP charging behavior,

2. The NP discharging behavior is not modeled at all,

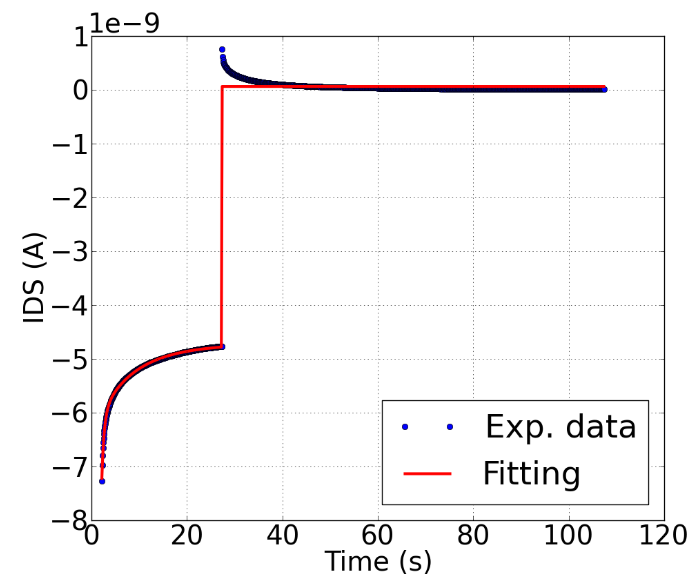

Fig. 4. Charging characterization of NOMFET.

Our hypothesis is that the NOMFET actually includes a network of many gold nanoparticles in its channel with a variety of time constants while our very simple model only consider first order time constant. The solution which were proposed to refine our model is to introduce a second order time constant $q_{2}$ in our model as described in equations (7)-(9):

$$
\begin{aligned}
& q_{1}=\left(q_{1}-q_{0}+C_{N P 1} V_{N P}\right) e^{-\left(d t / \tau_{C 1}\right)}+q_{0}-C_{N P 1} V_{N P}, \\
& q_{2}=\left(q_{2}-q_{0}+C_{N P 2} V_{N P}\right) e^{-\left(d t / \tau_{C 2}\right)}+q_{0}-C_{N P 2} V_{N P} .
\end{aligned}
$$

The charge final total is:

$$
q=q_{1}+q_{2}
$$


The introduction of this second time constant improves the modelling of the charge behavior but does not improve the one of the discharge. As shown in Fig. 4, when $I_{D S}$ return 0 after $V_{G S}$ has been set to zero of positive voltage (solid curve), it does not exhibit the characteristic discharge behavior shown in the experimental data (dots curve).

\section{III.3. Discharge modeling}

Figure 5 shows a simplified equivalent circuit for the NOMFET. With a negative voltage applied on the gate, the gold nanoparticles are charged as capacitors. When the voltage on the gate is zero or positive, NPs are discharged through the drain. In order to model the discharge dynamic, it is necessary to modify the basic formulation of the EKV model by introducing a term of discharge $\frac{d q}{d t}$ after the charge processing with two charge constants $C_{q 1}, C_{q 2}$ hence the current between the source and drain is calculated by

$$
I_{D S}=I_{S} \frac{I_{f o r}-I_{r e v}}{1+\theta V_{p}}+I_{o f f}-C_{q 1} \frac{d q 1}{d t}-C_{q 2} \frac{d q 2}{d t} .
$$

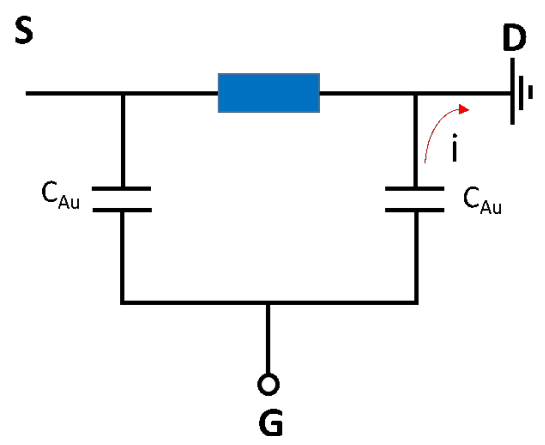

Fig. 5. The gold nanoparticle is like a capacitor.

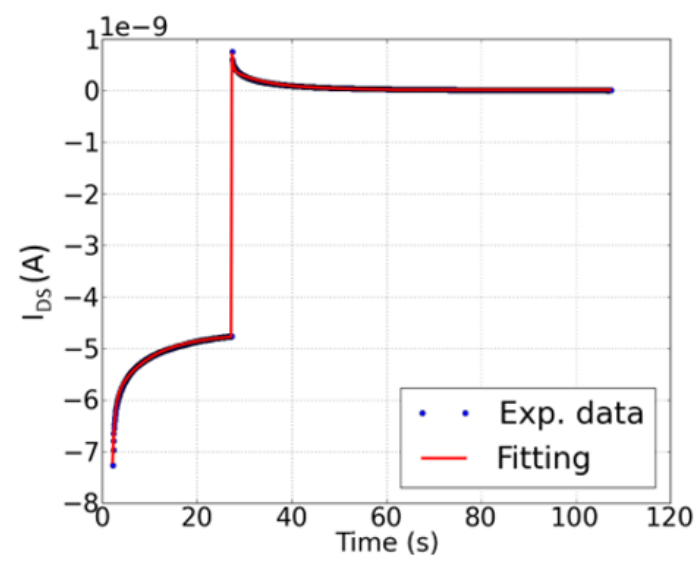

Fig. 6. Characterization of temporal function of NOMFET by EKV. 
Finally, by using this refinement, we obtain a good fitting of temporal dynamic for NOMFET device with experimental data as can be seen in Fig. 6.

\section{RESULTS AND DISCUSSION}

The iterative calculation of EKV model is programmed in Python language, which uses the least-square function and several useful libraries for fitting. The order of magnitude and the physical and/or mathematical constraints are specified for each variable. The initial values of the variables for the fitting are chosen in agreement with their order of magnitude, which can also be used to add additional boundaries to improve the convergence of the fitting.

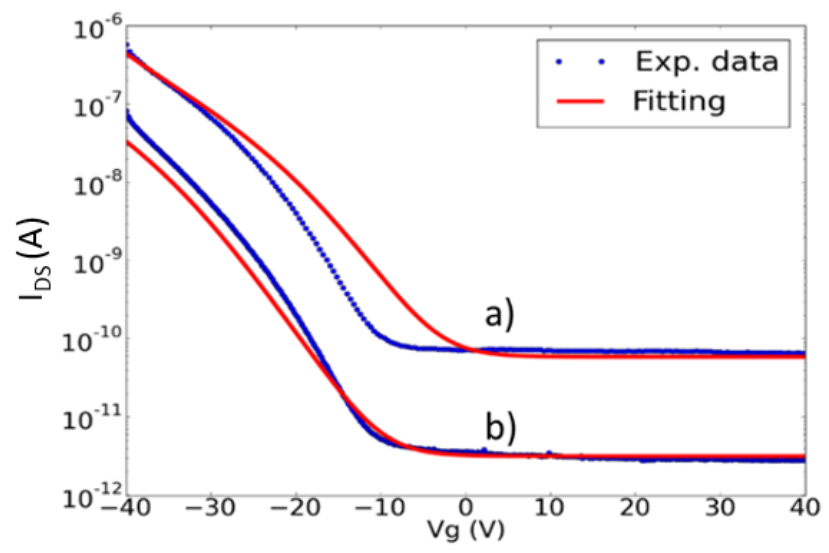

Fig. 7. The current $\mathrm{I}_{D S}$ in function of $\mathrm{V}_{G}$ : a) after erasing at $V=-50 \mathrm{~V}, \mathrm{~b}$ ) after writing at $V=+50 \mathrm{~V}$.

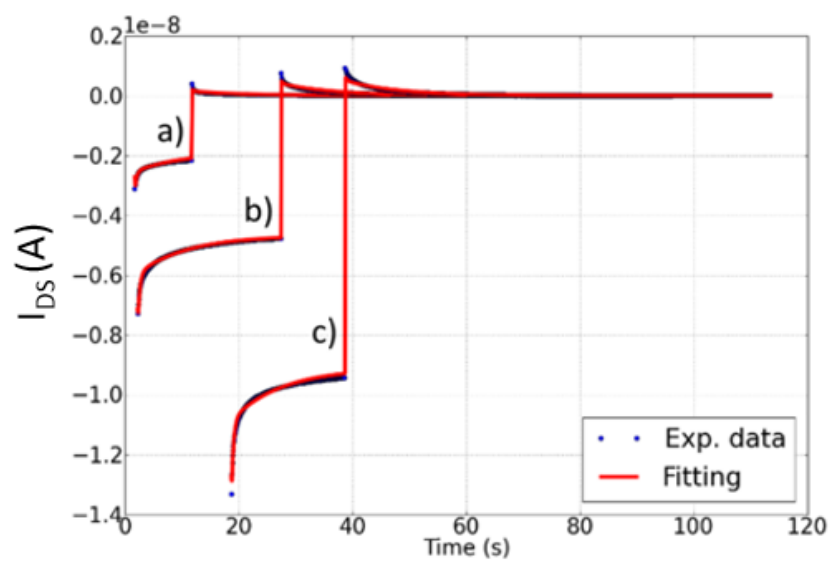

Fig. 8. Current $I_{D S}$ as a function of time for charge and discharge steps: a) $V_{G}=-20 \mathrm{~V}$; b) $V_{G}=-30 \mathrm{~V}$; c) $V_{G}=-40 \mathrm{~V}$.

Here, by using the global fitting, the EKV model integrates both two characterizations of memory-transistor and temporal dynamic of NOMFET with a series of applied voltage in the same 
time. The first results shown in Fig. 7 are obtained by measuring the current $I_{D S}$ as a function of the voltage applied on the gate $V_{G}$. Two sets of measurements are plotted: a) after a voltage of $-50 \mathrm{~V}$ has been applied for erasing and b) after writing at $V=+50 \mathrm{~V}$ for a read on the gate during $30 \mathrm{~s}$. We swept the gate voltage from $-40 \mathrm{~V}$ to $+40 \mathrm{~V}$. We observe a behavior that confirms the p-type semiconductor of our device: the current is increases with negative voltages, and is constant for positive voltages, we see good agreement between our model and the measurements.

The second result shown on Fig. 8 is simulated by using the same parameter of charge. We succeeded to fit 3 dynamic curves of the current for the charge and the discharge with increased negative voltages applied at the gate $-20 \mathrm{~V},-30 \mathrm{~V},-40 \mathrm{~V}$. The result shows a good agreement of our model with the dynamic behavior of the NOMFET for both the charge and discharge behaviors.

Table 1. Variable parameters of charge.

\begin{tabular}{clc}
\hline Symbol & Quantity & Transistor/temporal dynamic \\
\hline \hline $\mathrm{tc}_{1}$ & Time constant & $8.97 \mathrm{~s}$ \\
$\mathrm{tc}_{2}$ & Time constant & $0.54 \mathrm{~s}$ \\
$\mathrm{cNp}_{1}$ & Charge constant & $3.37-11 \mathrm{~F}$ \\
$\mathrm{cNp}_{2}$ & Charge constant & $7.75-11 \mathrm{~F}$ \\
$\mathrm{C}_{O X}$ & Capacitor of oxide & $7.01-10 \mathrm{~F} / \mathrm{m}$ \\
\hline
\end{tabular}

Table 2. Variable parameters of EKV model.

\begin{tabular}{llcc}
\hline Symbol & Quantity & Transistor & Temporal dynamic \\
\hline \hline is & Time constant & $-5.24^{-9} \mathrm{~A}$ & $-5.77^{-11} \mathrm{~A}$ \\
$\mathrm{vt}_{0}$ & Time constant & $-18 \cdot 86 \mathrm{~V}$ & $-2.03 \mathrm{~V}$ \\
$\mathrm{n}$ & Charge constant & 94.39 & 54.89 \\
theta & Charge constant & -3.72 & -0.15 \\
$\mathrm{i}_{\text {Off }}$ & Current in off- state & $-6.76^{-8} \mathrm{~A}$ & $5.01^{-11} \mathrm{~A}$ \\
$\mathrm{~S}$ & Subthreshold swing & 6.29 & 6.29 \\
\hline
\end{tabular}

Finally, our fitting parameters can be divided in two parameter groups, the parameters for charge, in Table 1, which are kept for all characterization of NOMFET and the parameters of transistor EKV model, in Table 2, which are chosen for each characterization. 


\section{CONCLUSION}

In this paper, we have developed the EKV model, a model which is based on fundamental physical considerations and relation between the current, charges and the voltage for the NOMFET. The EKV model requires a small number of parameters, simplifies implementation, hierarchical structure for characterization of NOMFET in both memory-transistor and temporal dynamic for a nano-device. By using the same parameters of charge, the fitting function can be reproduced for many experimental results, the techniques can be useful to study other memristive nanodevices and can show a good exploitation for application.

We have developed the EKV model refinement based parameters to specific, values; this allows an approach in the design of new circuits for bio-inspired devices. Modelling the NOMFET using a MOSFET model such as the EKV model provides a fair physical understanding of the device. The model is simple and compact enough to be used for the modelling of circuit architectures and most notably spiking neuromorphic circuit for which the modelling of dynamic properties is very important.

\section{ACKNOWLEDGMENT}

The authors would like to thank the European Union within the project Synaptic Molecular Networks for Bio-inspired Information Processing (SYMONE) and the French National Research Agency within the project Synapse-like Transistor and circuit for neuro-inspired computing architecture (SYNAPTOR) for supporting in part.

\section{REFERENCES}

[1] F. Alibart, S. Pleutin, O. Bichler, C. Gamrat, T. Serrano-Gotarredona, B. Linares-Barranco and D. Vuillaume, Advanced Functional Materials 22 (2012) 609.

[2] C. Novembre, D. Guerin, K. Lmimouni, C. Gamrat and D. Vuillaume, Appl. Phys. Lett. 92 (2008) 94.

[3] F. Alibart, S. Pleutin, D. Guérin, C. Novembre, S. Lenfant, K. Lmimouni, C. Gamrat and D. Vuillaume, Advanced Functional Materials 20 (2010) 330.

[4] O. Bichler, W. Zhao, F. Alibart, S. Pleutin, D. Vuillaume and C. Gamrat, IEEE Transactions on Electron Devices 57 (2010) 3115.

[5] G. Vicente-Sánchez, J. Velarde-Ramírez, T. Serrano-Gotarredona and B. Linares-Barranco, Int. J. Analog. Int. Circ. 59 (2009) 325.

[6] M. Tsodyks, K. Pawelzik and H. Markram, Neural Computation 10 (1998) 821.

[7] L. F. Abbott, J. Varela, K. Sen and S. Nelson, Science 275 (1997) 221.

[8] C. C. Enz, IEEE Solid-State Circuits Society Newsletter 13 (2008) 24.

[9] H. Oguey and S. Cserveny, Summer Course on" Process and Device Modelling," ESAT Leuven-Heverlee, Belgium (1983) .

[10] C. C. Enz, F. Krummenacher and E. A. Vittoz, Analog integrated circuits and signal processing 8 (1995) 83.

[11] T. Serrano-Gotarredona, B. Linares-Barranco, G. Agnus, V. Derycke, J. Bourgoin, F. Alibart, D. Vuillaume, J. Sohn, J. Bendall, M. Welland et al., Fast and compact simulation models for a variety of fet nano devices by the cmos ekv equations, Nanotechnology, 2009. IEEE-NANO 2009. 9th IEEE Conference on, IEEE, 2009, pp. 691-694.

[12] C. Enz, M. Bucher, A. Porret, J. Sallese and F. Krummenacher, The foundations of the ekv mos transistor chargebased model, Technical Proceedings of the 2002 International Conference on Modeling and Simulation of Microsystems, 2002.

[13] K.-C. Kwon, J.-S. Lee, C. G. Kim and J.-G. Park, Appl. Phys. Express 6 (2013) 067001.

[14] C. Hu, Modern semiconductor devices for integrated circuits, vol. 1, Prentice Hall Upper Saddle River, NJ, 2010. 